\title{
The flaring and quiescent components of the solar corona
}

\author{
C. Argiroffi ${ }^{1,2}$, G. Peres ${ }^{1,2}$, S. Orlando ${ }^{2}$, and F. Reale ${ }^{1,2}$ \\ 1 Dipartimento di Scienze Fisiche ed Astronomiche, Sezione di Astronomia, Università di Palermo, Piazza del Parlamento 1, \\ 90134 Palermo, Italy \\ e-mail: [argi; peres; reale] @astropa.unipa.it \\ 2 INAF - Osservatorio Astronomico di Palermo, Piazza del Parlamento 1, 90134 Palermo, Italy \\ e-mail: orlando@astropa.inaf.it
}

Received 5 January 2008 / Accepted 30 April 2008

\section{ABSTRACT}

\begin{abstract}
Context. The solar corona is a template to understand stellar activity. The Sun is a moderately active star, and its corona differs from that of active stars: for instance, active stellar coronae have a double-peaked emission measure distribution $E M(T)$ with a hot peak at 8-20 MK, while the non-flaring solar corona has one peak at 1-2 MK and, typically, much cooler plasma.

Aims. We study the average contribution of flares to the solar emission measure distribution to investigate indirectly the hypothesis that the hot peak in the $E M(T)$ of active stellar coronae is due to a large number of unresolved solar-like flares, and to infer properties about the flare distribution from nano- to macro-flares.

Methods. We measure the disk-integrated time-averaged emission measure, $E M_{\mathrm{F}}(T)$, of an unbiased sample of solar flares, analyzing uninterrupted GOES/XRS light curves over time intervals of one month. We obtain the $E M_{\mathrm{Q}}(T)$ of quiescent corona for the same time intervals from Yohkoh/SXT data. To investigate how $E M_{\mathrm{F}}(T)$ and $E M_{\mathrm{Q}}(T)$ vary during the solar cycle, we evaluate them at different phases of the cycle between December 1991 and April 1998.

Results. Irrespective of the solar cycle phase, $E M_{\mathrm{F}}(T)$ appears as a peak in the distribution, and it is significantly larger than the values of $E M_{\mathrm{Q}}(T)$ for $T \sim 5-10 \mathrm{MK}$. As a result, the time-averaged $E M(T)$ of the entire solar corona is double-peaked, with the hot peak, due to time-averaged flares, being located at temperatures similar to those of active stars, but less enhanced. The $E M_{\mathrm{F}}(T)$ shape supports the hypothesis that the hot $E M(T)$ peak of active coronae is due to unresolved solar-like flares. If this is the case, quiescent and flare components should follow different scaling laws for increasing stellar activity. In the assumption that the heating of the corona is entirely due to flares, from nano- to macro-flares, then either the flare distribution or the confined plasma response to flares, or both, are bimodal.
\end{abstract}

Key words. stars: activity - stars: coronae - Sun: activity - Sun: corona - Sun: flares - Sun: X-rays, gamma rays

\section{Introduction}

Late-type stars emit X-rays from their coronae, which consist of hot plasma $(T \sim 1-20 \mathrm{MK})$ magnetically confined in the outer atmosphere. Stellar coronae have a wide variety of X-ray luminosities: from $\sim 10^{26}-10^{27} \mathrm{erg} \mathrm{s}^{-1}$ in low-activity stars, including the Sun, to $\sim 10^{31} \mathrm{erg} \mathrm{s}^{-1}$ in very active stars (see Favata \& Micela 2003; Güdel 2004, and references therein). The physical differences between the coronae of stars of different activity levels are debated.

To explain the higher X-ray luminosities, it was proposed that active stars could have large surface coverage of solar-like coronal structures (Walter et al. 1980). However this hypothesis does not agree with recent findings. In fact, active stars have non-uniform and incomplete surface coverage, such as the Sun, as demonstrated by various observational measurements: X-ray rotational modulation (Güdel et al. 1995; Marino et al. 2003); small coronal volume inferred from measured plasma densities (Testa et al. 2004a; Ness et al. 2004), and from resonance scattering (Testa et al. 2004b, 2007). Moreover, even a complete surface coverage of solar-like non-flaring coronal structures cannot explain the X-ray emission level of high-activity stars (Drake et al. 2000; Peres et al. 2004).

Active stellar coronae differ from that of the Sun also in terms of the average plasma temperatures. The emission measure distribution vs. temperature, $E M(T)^{1}$ of active stellar coronae is usually peaked around $\sim 8-20 \mathrm{MK}$, and, in some cases, with large amounts of emission measure at very high temperatures $(T \sim 20-30 \mathrm{MK}$, Güdel et al. 1997; Sanz-Forcada et al. 2002, 2003; Scelsi et al. 2005). The hot bump in the $E M(T)$ of active stars was observed also in the absence of evident flaring activity, i.e. a time-independent emission (e.g. Sanz-Forcada \& Micela 2002; Argiroffi et al. 2003). Conversely, for the Sun and less active stars, the $E M(T)$ peaks at cooler temperatures ( $T \sim 1-2 \mathrm{MK}$, Peres et al. 2000; Raassen et al. 2003).

For the Sun, significant amounts of emitting plasma at very high temperatures is observed only during flares (e.g. Reale et al. 2001). It was therefore suggested that the $\sim 8-20 \mathrm{MK}$ peak in the $E M(T)$ of active stars, could be the result of a large number of unresolved solar-like flares (e.g. Güdel et al. 1997). However, this hypothesis cannot be tested directly because a large number of solar-like flares would produce light curves indistinguishable from those corresponding to a steady emission for the combined effect of flare superposition and available $S / N$ ratio.

Since the hot bump in the $E M(T)$ of active stars may be due to an average process over the stellar disk and observing time, we explore the hypothesis that the Sun may show such a bump

$1 E M(T)$, used to characterize optically thin hot plasmas, is defined as the amount of emission measure, $N_{\mathrm{e}}^{2} \Delta V$, of plasma with temperature ranging from $T-\Delta T / 2$ to $T+\Delta T / 2$. 
when observed full disk and by averaging over a sufficiently long time; we also test the hypothesis that the $\sim 8-20 \mathrm{MK}$ peak in the $E M(T)$ of active stars is the result of a large number of solarlike flares. To this end, we derive the averaged $E M(T)$ of a large number of flares in the solar corona, and compare this with the hot peak in the active coronae $E M(T)$.

This study allows us check whether the reason for the absence of a double peak in the solar $E M(T)$ is that solar flares are rather sparse and that they are observed with different methods and observing modes than the quiescent solar corona and active stars. At the same time, we may demonstrate a link between the solar and stellar coronae.

The work presented in this paper partly follows the approach of Orlando et al. (2000) and Peres et al. (2000). They analyzed data gathered with the Soft X-ray Telescope (SXT, Tsuneta et al. 1991), onboard the Yohkoh satellite, to derive the $E M(T)$ of the solar corona including both the quiescent components (quiet corona, active regions, active region cores, Orlando et al. 2001, 2004), and individual flares (Reale et al. 2001).

The Yohkoh/SXT normally operates in quiet mode, during which the entire solar corona is observed. An abrupt increase in count rate prompts the instrument to change into the flareobserving mode: only the flaring region is then monitored with a high sampling cadence. When the count rate decreases, the satellite switches again to quiet mode. Therefore, the flare mode is dedicated to portions of the solar corona for limited time intervals. As a consequence, the Yohkoh/SXT flare observations, on the one hand, miss information about the entire disk coronal emission, and on the other, lack long and continuous time coverage, and therefore do not allow an estimation of the average $\operatorname{EM}(T)$ of all solar flares. To fill this gap, we analyzed data gathered by the X-ray Sensor (XRS) photometers (Garcia 1994) onboard the GOES satellites. The GOES/XRS provides continuous disk-integrated flux of the solar corona in two different X-ray bands with a time resolution of $\delta t \sim 3 \mathrm{~s}$. We devised a method to derive time-averaged flaring $E M(T)$ distributions using this disk-integrated data.

We analyzed GOES/XRS data over long uninterrupted time intervals, to include a representative and large set of detectable solar flares, and hence to derive an average $E M(T)$ of solar flares. We use this average flaring $E M(T)$ of the Sun to interpret those of active stars in terms of stellar flares. This hypothesis relies on the assumption that the set of phenomena occurring in the Sun are to some extent equivalent to that occurring in more active stars on shorter timescales; thus, time-averaging over an adequately long time interval would produce results similar to the scenario derived from disk-integrated time-averaged stellar observations.

The derivation of both the average flaring $E M(T)$ and quiescent $E M(T)$ (Peres et al. 2000), enables, for the first time, the evaluation of a total $E M(T)$, which models both the quiescent corona and flares. The estimation of the total $E M(T)$ is important also for investigating the coronal heating mechanism (e.g. Cargill 1994). Parker (1988) suggested that coronal plasma could be heated entirely by a large number of small magnetic reconnections. This idea relies on the observed frequency distribution of flares versus energy: many studies showed that $\mathrm{d} N / \mathrm{d} E \propto E^{-\alpha}$, with $\alpha \sim 2$ (e.g. Hudson 1991). The total amount of energy deposited by flares in the corona depends on the maximum and minimum flare energy and on the exact value of $\alpha$. Therefore, given reasonable values of these quantities, the high temperature coronal plasmas could be attributed to the energy release of flares. For a given corona model of fixed heating function and loop geometry, the temperature structure is entirely constrained (plasma cooling via conduction or radiation is known), and hence also the $E M(T)$. Therefore, different heating mechanisms predict different $E M(T)$, and the observed $E M(T)$ is a key test for any coronal heating model (Cargill 1994).

In Sect. 2, we present the method applied to derive the average $E M(T)$ of flaring and quiescent plasma in the solar corona from GOES/XRS and Yohkoh/SXT data, and the crosscalibration between these two instruments. The derived results are reported in Sect. 3 and discussed in Sect. 4.

\section{Method}

Hereafter we refer to as the quiescent corona that observed in the full disk images of Yohkoh/SXT, when individual flares are either absent or, if present, very weak, and the total emission is characterized by variations on long time scales (days). On the other hand, we refer to as flaring emission the part of emission due to major flares, i.e. identified in GOES/XRS light curves.

\subsection{Flaring $\operatorname{EM}(T)$}

To obtain the flaring $\operatorname{EM}(T)$, we analyzed the disk-integrated $\mathrm{X}$-ray light curves recorded by $G O E S / \mathrm{XRS}$, because these data provided a complete time coverage of solar flares and a complete sample of events, at variance with the Yohkoh/SXT data set. We analyzed long time intervals of GOES/XRS data to recover representative samples of solar flares. We considered time intervals of one month because they are:

- significantly longer than the duration of individual solar flares (from a few minutes to a few hours);

- sufficiently long to encompass a significant number of flares;

- sufficiently long to provide a survey of all the solar surface and its magnetic field, and hence avoid effects due to rotational modulation;

- sufficiently short to enable any variations during the longterm evolution of the solar corona not to be removed by averaging of signal and, at the same time, allow us to repeat the analysis at different phases of the solar cycle to explore variations in the flare component.

GOES/XRS data consist of disk-integrated solar flux gathered in two bands, $1-8 \AA$ and $0.5-4.0 \AA$, with a time resolution of $\delta t \sim 3 \mathrm{~s}$. Since we planned to derive only the characteristics of the flaring plasma, we evaluated and subtracted the contribution of the quiescent corona to the observed fluxes. For an individual flare analysis, such a contribution is the preflare flux in each band. This may not be accurate for long time periods, because both the soft and hard light curves include the superposition of many flares, especially close to the maximum of the solar cycle. Hence, we assumed that the quiescent fluxes in the two bands are the minimum flux values in the $24 \mathrm{~h}$ time interval. Details about the quiescent contribution estimation are given in Appendix A. In Fig. 1, we show, as an example, $24 \mathrm{~h}$ GOES/XRS light curves whose minimum levels represent quiescent emission.

For each time bin $\delta t$, we derived the flaring plasma temperature $T$ and the flaring plasma $E M$, applying the flux ratio method (e.g. Garcia 1994) to the background-subtracted fluxes. This method assumes that the flaring plasma is isothermal during the time interval $\delta t$. The derived $T$ are an average weighted for the emission measure of all flaring plasma observed during that time interval. Hence, we obtained a one-month time sequence of $T$ and $E M$ for the total flaring corona.

To obtain a time-averaged $E M(T)$ distribution, we multiplied each $E M$ value for the duration of its time bin $\delta t$ and divided by 


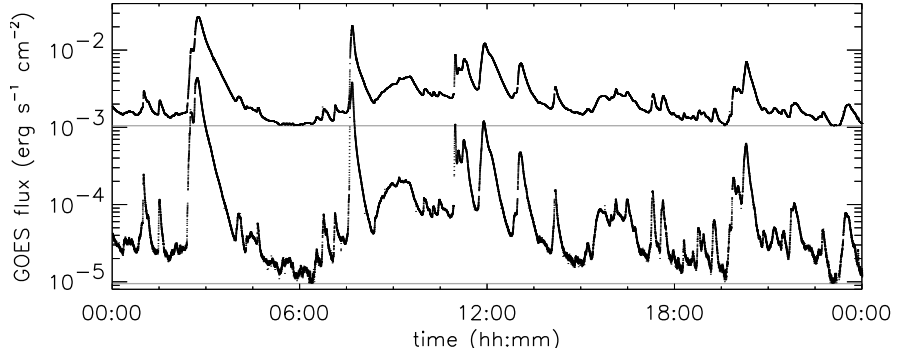

Fig. 1. GOES/XRS light curves in the soft and hard bands registered on December 5, 1991. Gray lines mark the minimum emission in the two bands for this $24 \mathrm{~h}$ interval.

the duration $\Delta t$ of the entire month, i.e. $\overline{E M}=E M \delta t / \Delta t$. Güdel et al. (1997) applied the same approach to the time average for a single flare.

We considered the same temperature grid adopted for the Yohkoh/SXT analysis (see Sect. 2.2). This grid includes the nominal temperature sensitivity of GOES/XRS, and its binning is appropriate for the typical temperature accuracy.

We then summed all $\overline{E M}$ values whose temperatures were in the same temperature bin. Applying this procedure, we obtained a time-averaged $E M(T)$ of the solar flare component (hereafter indicated as $\left.E M_{\mathrm{F}}(T)\right)$.

\subsection{Quiescent $E M(T)$}

We determined the quiescent $E M(T)$ of the entire Sun by applying the Peres et al. (2000) method to the Yohkoh/SXT images. In the normal observing mode, the Yohkoh/SXT acquires images of the entire Sun in different filters. We derived the $E M(T)$ from images obtained using the Al.1 and AlMg filters. We constructed a $E M$ versus $T$ histogram for the quiescent corona using the $E M$ and $T$ maps obtained with the filter ratio method (Vaiana et al. 1973), based on the assumption that the plasma within each pixel is isothermal. We adopted the same temperature grid as Peres et al. (2000), i.e. a grid ranging from 0.3 to $100 \mathrm{MK}$ equally spaced on a logarithmic scale. To obtain monthaveraged $E M(T)$ (hereafter indicated as $E M_{\mathrm{Q}}(T)$ ) of the slowlychanging quiescent solar corona, we averaged four $E M(T)$ of the quiescent corona separated by about a week.

Yohkoh/SXT observations were selected such that they included images of the entire solar corona in two filters (Al.1 and $\mathrm{AlMg}$ ), with two exposure times (short and long), for a total of four images separated by at most a few minutes (see Orlando et al. 2000, for more details).

\subsection{Cross calibration between Yohkoh/SXT and GOES/XRS data}

We cross-calibrated Yohkoh/SXT and GOES/XRS, to be able to compare and analyze jointly the quiescent and flare emission measure distribution, $E M_{\mathrm{Q}}(T)$ and $E M_{\mathrm{F}}(T)$, derived from these two instruments. We selected a sample of flares, listed in Table 1: a subset of the flares analyzed by Reale et al. $(2001)^{2}$, and the so-called Masuda flare (Masuda et al. 1994). We analyzed the data on these flares collected by both the instruments. This data set studies a large range of flare intensity, from weak (GOES

\footnotetext{
${ }^{2}$ We discarded the X9 flare of the Reale et al. set because it was oversampled with respect to the other flares, otherwise its weight in the cross-calibration would be too high.
}

Table 1. Flares analyzed for the Yohkoh vs. GOES cross-calibration.

\begin{tabular}{cccccc}
\hline \hline N. & Date & Class & N. & Date & Class \\
\hline $1^{a}$ & 18 Jul. 1992 & C5.8 & $5^{a}$ & 27 Feb. 1994 & M2.8 \\
$2^{a}$ & 10 Feb. 1993 & M1.0 & $6^{a}$ & 26 Dec. 1991 & M4.2 \\
$3^{a}$ & 19 Oct. 1992 & M1.1 & $7^{a}$ & 06 Feb. 1992 & M7.6 \\
$4^{b}$ & 13 Jan. 1992 & M2.0 & $8^{a}$ & 15 Nov. 1991 & X1.5 \\
\hline
\end{tabular}

${ }^{a}$ Flares analyzed by Reale et al. (2001). ${ }^{b}$ Masuda flare.

class $\mathrm{C} 5.8$ ) to very intense (X1.5), enabling an appropriate crosscalibration to be made.

We derived the $T$ and $E M$ time sequence from the Yohkoh/SXT data, following the approach of Reale et al. (2001). The Yohkoh/SXT flares were observed in flare mode. Each data set consisted of a sequence of frames acquired alternatively using the Be $119 \mu \mathrm{m}$ and $\mathrm{Al} 11.4 \mu \mathrm{m}$ filters, with a time sampling that ranged between $\sim 10 \mathrm{~s}$ and $\sim 100 \mathrm{~s}$. Each frame is composed of images containing $64 \times 64$ pixels of 2 ". 5 side. From each pair of filter images, we derived $E M$ and $T$ maps, from which we computed the $E M$-weighted average temperature and total emission measure.

We derived the simultaneous $E M$ and $T$ values from the GOES/XRS data as described in Sect. 2.1. We subtracted the flux values in the two bands just before the flare start, assuming that during the flare evolution the background coronal emission remained constant. From the GOES/XRS flare analysis, we obtained a time series of $T$ and $E M$ for each flare. Since the time sampling of Yohkoh/SXT data is less frequent than that of $G O E S / \mathrm{XRS}$, we considered the $E M$ and $T$ values given by the GOES/XRS data at the times of the Yohkoh/SXT data.

The derived results of $T$ and $E M$ evolution for each flare from Yohkoh/SXT and GOES/XRS are shown in Fig. 2. The temperatures derived using the GOES/XRS data are in general higher than those measured using Yohkoh/SXT data, especially for the more energetic flares. The opposite occurs for the emission measure. Figure 3 shows the correspondence between the values of temperature and emission measure derived from GOES/XRS and Yohkoh/SXT data, indicated by $T_{\mathrm{G}}, T_{\mathrm{Y}}$, $E M_{\mathrm{G}}, E M_{\mathrm{Y}}$. For the cross-calibration of temperature and emission measure, we assumed a power-law function. The best-fit functions were:

$T_{\mathrm{Y}}=a_{1} T_{\mathrm{G}}^{a_{2}} \quad E M_{\mathrm{Y}}=a_{3} E M_{\mathrm{G}}^{a_{4}}$

with $a_{1}=3.06 \pm 0.01, a_{2}=0.491 \pm 0.002, a_{3}=0.520 \pm 0.002$, $a_{4}=1.1010 \pm 0.0003$, with temperatures in units of MK, and emission measure values in units of $10^{44} \mathrm{~cm}^{-3}$. The best-fit functions, which reproduced the observed pattern well, were used to correct $T$ and $E M$ values derived from the GOES/XRS data.

The scatter between $T_{\mathrm{Y}}$ values, corresponding to similar $T_{\mathrm{G}}$ values, is significantly larger than the typical $T_{\mathrm{Y}}$ error bar (see Fig. 3). This implies that the uncertainty in the inferred $T_{\mathrm{Y}}$ values is provided by the scatter in the observed points. This is also the case for the emission measure. The observed scatter is $5 \%$ $(1 \sigma)$ for $T_{\mathrm{Y}}$ and $20 \%(1 \sigma)$ for $E M_{\mathrm{Y}}$. The bin widths of the temperature grid over which we constructed the emission measure distributions are comparable to the uncertainties in the derived temperatures.

\section{Data sets and results}

We derived the emission measure distributions, $E M_{\mathrm{Q}}(T)$ and $E M_{\mathrm{F}}(T)$, of the quiescent and flaring plasma of the solar corona averaged over one month. Since solar coronal characteristics, 

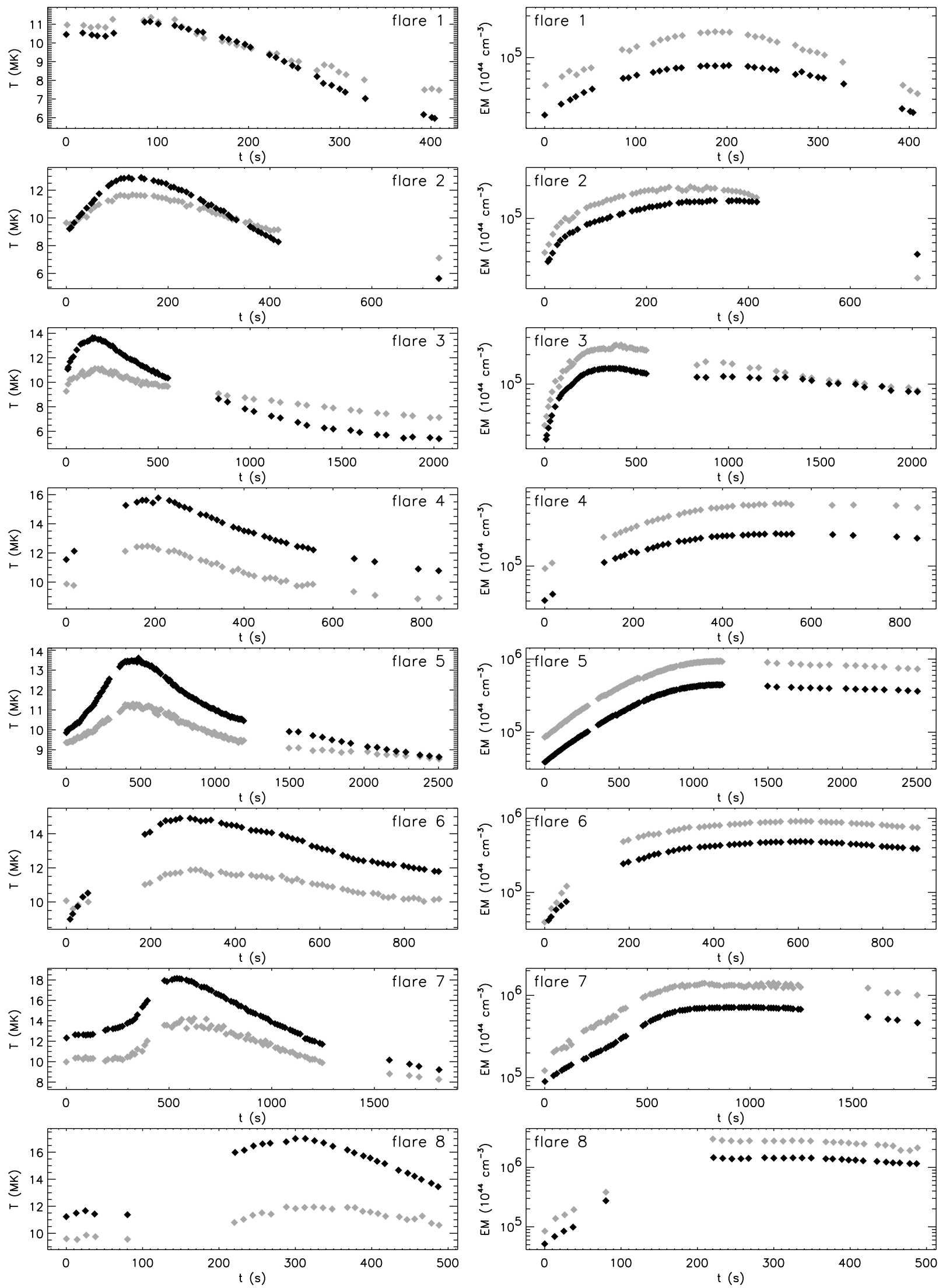

Fig. 2. Time series of temperature and total emission measure of the eight flares (see Table 1) analyzed for the cross calibration between GOES/XRS and Yohkoh/SXT. Black and gray symbols mark the GOES/XRS and Yohkoh/SXT data respectively. 

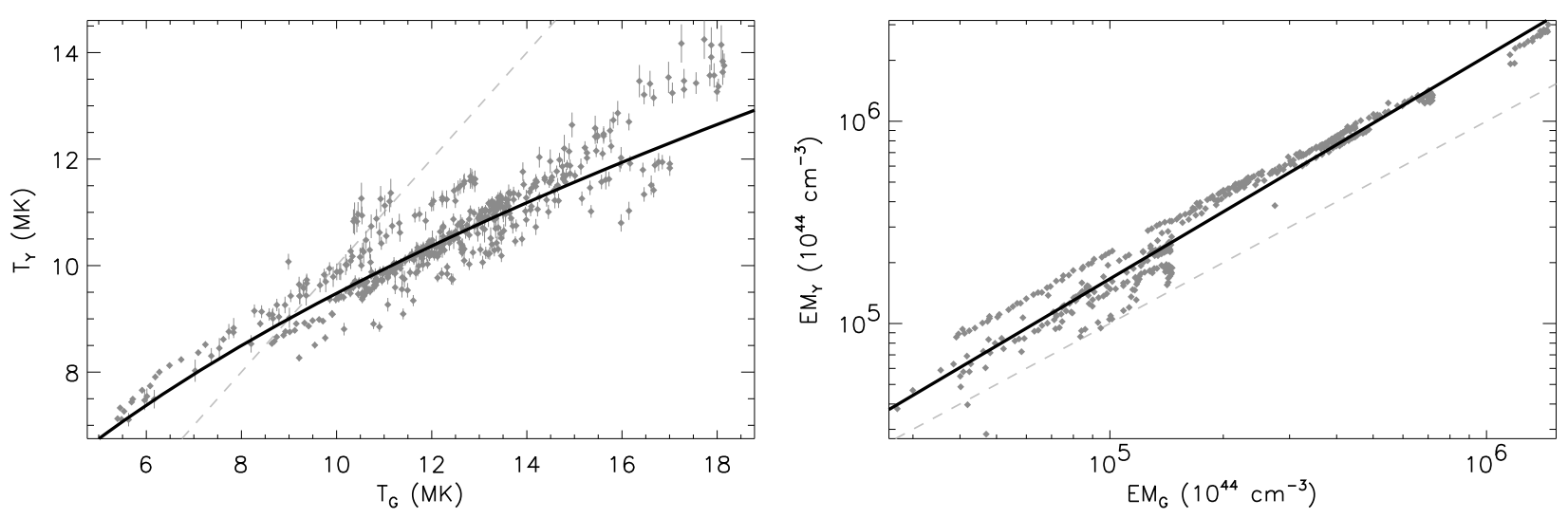

Fig. 3. Flare temperatures (left panel) and emission measure (right panel) derived from the Yohkoh/SXT and GOES/XRS data for the flares listed in Table 1. Solid lines indicate the best fit functions, dashed lines the bisectors.

(i.e. total emission measure, average temperature, flare intensity, and frequency) change considerably during the solar cycle, we repeated the derivation of $E M_{\mathrm{Q}}(T)$ and $E M_{\mathrm{F}}(T)$ at different stages of the solar cycle to probe their dependence on the solar activity level.

We measured the flaring and quiescent $\operatorname{EM}(T)$ every four months, obtaining a total of 20 analyzed months between December 1991 and April 1998, i.e. including both the maximum and minimum phases of the solar cycle. All dates of Yohkoh/SXT and GOES/XRS observations for each analyzed month are listed in Table 2. Figure 4 shows the $E M_{\mathrm{Q}}(T)$ and $E M_{\mathrm{F}}(T)$, derived for all the inspected months.

The quiescent component - whose shape and variations during the solar cycle were studied by Peres et al. (2000) - displays a peak at $T \sim 1-2 \mathrm{MK}$, and a gradual decrease at higher temperature. $E M_{\mathrm{Q}}(T)$ changes during the solar cycle: close to minimum it is lower than close to maximum, its peak is located at cooler temperatures, and its slope at high temperature is steeper.

The first noticeable result is that the $\operatorname{EM}(T)$ of the flare component is narrow and peaks at $T \sim 6-8 \mathrm{MK}$. This peak is significantly higher than the level of the quiescent plasma in the same temperature range, and is present independently of the phase of the solar cycle. The $E M_{\mathrm{F}}(T)$ height follows the solar cycle: it is higher at solar maximum than at minimum by a factor $\sim 10^{2}$. In contrast, both the peak temperature and $E M$-averaged temperature of the flare component do not show clear trends with the phase of the solar cycle. Hence, the amount of flaring emission measure varies largely across the solar cycle, while its average temperature does not.

By summing $E M_{\mathrm{F}}(T)$ and $E M_{\mathrm{Q}}(T)$, we obtained the total $E M(T)$ of entire solar corona, including the average effect of both flare and quiescent plasma (see Fig. 4). The quiescent component dominates at low temperatures, while the flaring $E M_{\mathrm{F}}(T)$ dominates for $T \sim 5-10 \mathrm{MK}$. At higher temperatures $(T \geq 20 \mathrm{MK}), E M_{\mathrm{Q}}(T)$ is larger than $E M_{\mathrm{F}}(T)$. However, results for such high temperatures must be considered with caution because the two Yohkoh/SXT filters used for deriving $E M_{\mathrm{Q}}(T)$ are not tailored for hot plasma (Orlando et al. 2000). In summary, the total $\operatorname{EM}(T)$ of the entire solar corona is double peaked, with the cooler peak, at $T \sim 1-2 \mathrm{MK}$ related to the quiescent corona, and the hotter peak, at $T \sim 6-8 \mathrm{MK}$, to the flares.

The variations during the solar cycle in the total amount of both quiescent and flaring emission measure (hereafter $E M_{\mathrm{O}}^{\mathrm{TOT}}$ and $E M_{\mathrm{F}}^{\mathrm{TOT}}$ ) are shown in Fig. 5. For both $E M_{\mathrm{Q}}^{\mathrm{TOT}}$ and $E M_{\mathrm{F}}^{\mathrm{TOT}}$, the observed uncertainties are far smaller than the size of the
Table 2. Yohkoh/SXT and GOES/XRS data.

\begin{tabular}{|c|c|c|c|c|c|c|}
\hline $\begin{array}{c}\text { Analyzed } \\
\text { month }\end{array}$ & \multicolumn{2}{|c|}{$\begin{array}{c}\text { GOES/XRS } \\
\text { start and stop date } \\
(\mathrm{dd} / \mathrm{mm} / \mathrm{yy} \mathrm{hh}: \mathrm{mm})\end{array}$} & \multicolumn{4}{|c|}{$\begin{array}{c}\text { Yohkoh/SXT } \\
\text { obs. 1, 2, 3, and } 4 \\
\text { (dd/mm/yy hh:mm) }\end{array}$} \\
\hline \multirow[t]{2}{*}{$\begin{array}{ll}\text { Dec. } & 1991\end{array}$} & start: $01 / 12 / 91$ & $00: 00$ & $03 / 12 / 91$ & $01: 35$ & $09 / 12 / 91$ & $07: 25$ \\
\hline & stop: $01 / 01 / 92$ & 00:00 & $16 / 12 / 91$ & $07: 15$ & $23 / 12 / 91$ & $23: 13$ \\
\hline \multirow[t]{2}{*}{$\begin{array}{ll}\text { Apr. } & 1992\end{array}$} & start: $01 / 04 / 92$ & $00: 00$ & $04 / 04 / 92$ & $01: 16$ & $11 / 04 / 92$ & $03: 54$ \\
\hline & stop: $01 / 05 / 92$ & 00:00 & $18 / 04 / 92$ & 03:21 & $25 / 04 / 92$ & $23: 53$ \\
\hline \multirow[t]{2}{*}{$\begin{array}{ll}\text { Aug. } & 1992\end{array}$} & start: $01 / 08 / 92$ & $00: 00$ & $05 / 08 / 92$ & $01: 34$ & $12 / 08 / 92$ & $23: 35$ \\
\hline & stop: $01 / 09 / 92$ & 00:00 & $19 / 08 / 92$ & 01:46 & $26 / 08 / 92$ & 01:04 \\
\hline \multirow[t]{2}{*}{$\begin{array}{ll}\text { Dec. } 1992 \\
\end{array}$} & start: $01 / 12 / 92$ & $00: 00$ & $05 / 12 / 92$ & $01: 49$ & $12 / 12 / 92$ & $04: 19$ \\
\hline & stop: $01 / 01 / 93$ & 00:00 & $19 / 12 / 92$ & $00: 16$ & $28 / 12 / 92$ & $14: 47$ \\
\hline \multirow[t]{2}{*}{ Apr. 1993} & start: $01 / 04 / 93$ & $00: 00$ & $05 / 04 / 93$ & $01: 41$ & $14 / 04 / 93$ & $21: 01$ \\
\hline & stop: $01 / 05 / 93$ & 00:00 & $19 / 04 / 93$ & $01: 37$ & $26 / 04 / 93$ & 00:44 \\
\hline \multirow{2}{*}{ Aug. 1993} & start: $01 / 08 / 93$ & $00: 00$ & $05 / 08 / 93$ & $03: 56$ & $12 / 08 / 93$ & $04: 39$ \\
\hline & stop: $01 / 09 / 93$ & 00:00 & $19 / 08 / 93$ & 00:29 & $26 / 08 / 93$ & 09:17 \\
\hline \multirow[t]{2}{*}{ Dec. 1993} & start: $01 / 12 / 93$ & $00: 00$ & $05 / 12 / 93$ & $15: 17$ & $12 / 12 / 93$ & $12: 41$ \\
\hline & stop: $01 / 01 / 94$ & 00:00 & $19 / 12 / 93$ & $05: 15$ & $26 / 12 / 93$ & $02: 38$ \\
\hline \multirow[t]{2}{*}{$\begin{array}{ll}\text { Apr. } & 1994\end{array}$} & start: 01/04/94 & $00: 00$ & $04 / 04 / 94$ & $23: 37$ & $12 / 04 / 94$ & $01: 51$ \\
\hline & stop: $01 / 05 / 94$ & 00:00 & $18 / 04 / 94$ & $23: 14$ & $25 / 04 / 94$ & $23: 52$ \\
\hline \multirow[t]{2}{*}{$\begin{array}{ll}\text { Aug. } & 1994\end{array}$} & start: $01 / 08 / 94$ & $00: 00$ & $05 / 08 / 94$ & 03:04 & $11 / 08 / 94$ & $22: 46$ \\
\hline & stop: $01 / 09 / 94$ & 00:00 & 19/08/94 & $17: 11$ & $26 / 08 / 94$ & 04:54 \\
\hline \multirow[t]{2}{*}{$\begin{array}{ll}\text { Dec. } & 1994\end{array}$} & start: $05 / 12 / 94$ & $00: 00$ & $07 / 12 / 94$ & $21: 23$ & $15 / 12 / 94$ & $23: 52$ \\
\hline & stop: $01 / 01 / 95$ & 00:00 & $20 / 12 / 94$ & $02: 43$ & $27 / 12 / 94$ & $17: 56$ \\
\hline \multirow[t]{2}{*}{$\begin{array}{ll}\text { Apr. } & 1995\end{array}$} & start: $01 / 04 / 95$ & $00: 00$ & $04 / 04 / 95$ & $06: 37$ & $11 / 04 / 95$ & $15: 18$ \\
\hline & stop: $01 / 05 / 95$ & 00:00 & $18 / 04 / 95$ & $12: 36$ & $24 / 04 / 95$ & 01:28 \\
\hline \multirow[t]{2}{*}{ Aug. 1995} & start: $01 / 08 / 95$ & $00: 00$ & $06 / 08 / 95$ & $12: 42$ & $13 / 08 / 95$ & $05: 13$ \\
\hline & stop: $01 / 09 / 95$ & 00:00 & $19 / 08 / 95$ & $02: 16$ & $25 / 08 / 95$ & $23: 34$ \\
\hline \multirow{2}{*}{ Dec. 1995} & start: $01 / 12 / 95$ & $00: 00$ & $05 / 12 / 95$ & $07: 58$ & $12 / 12 / 95$ & $13: 18$ \\
\hline & stop: $31 / 12 / 95$ & 00:00 & $19 / 12 / 95$ & $20: 27$ & $25 / 12 / 95$ & $23: 59$ \\
\hline \multirow[t]{2}{*}{ Apr. 1996} & start: $01 / 04 / 96$ & $00: 00$ & $05 / 04 / 96$ & $01: 45$ & $12 / 04 / 96$ & $11: 56$ \\
\hline & stop: $01 / 05 / 96$ & 00:00 & $19 / 04 / 96$ & $12: 27$ & $24 / 04 / 96$ & $23: 45$ \\
\hline \multirow[t]{2}{*}{$\begin{array}{ll}\text { Aug. } 1996\end{array}$} & start: $01 / 08 / 96$ & $00: 00$ & $06 / 08 / 96$ & $13: 22$ & $14 / 08 / 96$ & $10: 56$ \\
\hline & stop: $01 / 09 / 96$ & 00:00 & $21 / 08 / 96$ & 21:08 & $29 / 08 / 96$ & $10: 36$ \\
\hline \multirow[t]{2}{*}{$\begin{array}{ll}\text { Dec. } & 1996\end{array}$} & start: $01 / 12 / 96$ & $00: 00$ & $03 / 12 / 96$ & $18: 32$ & $13 / 12 / 96$ & $02: 15$ \\
\hline & stop: $31 / 12 / 96$ & 00:00 & $20 / 12 / 96$ & 14:04 & $27 / 12 / 96$ & 03:12 \\
\hline \multirow[t]{2}{*}{$\begin{array}{ll}\text { Apr. } & 1997\end{array}$} & start: $01 / 04 / 97$ & $00: 00$ & $04 / 04 / 97$ & $00: 14$ & $11 / 04 / 97$ & $07: 14$ \\
\hline & stop: $30 / 04 / 97$ & 00:00 & $18 / 04 / 97$ & $15: 51$ & $25 / 04 / 97$ & 03:21 \\
\hline \multirow[t]{2}{*}{$\begin{array}{ll}\text { Aug. } 1997\end{array}$} & start: $01 / 08 / 97$ & $00: 00$ & $05 / 08 / 97$ & $17: 38$ & $11 / 08 / 97$ & $22: 38$ \\
\hline & stop: $01 / 09 / 97$ & 00:00 & $18 / 08 / 97$ & 08:31 & $25 / 08 / 97$ & 00:51 \\
\hline \multirow{2}{*}{ Dec. 1997} & start: $01 / 12 / 97$ & $00: 00$ & $05 / 12 / 97$ & $00: 22$ & $12 / 12 / 97$ & $07: 15$ \\
\hline & stop: $01 / 01 / 98$ & 00:00 & $19 / 12 / 97$ & $17: 23$ & $26 / 12 / 97$ & $06: 27$ \\
\hline \multirow[t]{2}{*}{$\begin{array}{ll}\text { Apr. } & 1998\end{array}$} & start: $01 / 04 / 98$ & $00: 00$ & $05 / 04 / 98$ & $22: 41$ & $13 / 04 / 98$ & $13: 39$ \\
\hline & stop: $01 / 05 / 98$ & 00:00 & $18 / 04 / 98$ & 00:30 & $25 / 04 / 98$ & 17:06 \\
\hline
\end{tabular}

plotting symbols. In Fig. 5, both $E M_{\mathrm{Q}}^{\mathrm{TOT}}$ and $E M_{\mathrm{F}}^{\mathrm{TOT}}$ follow the solar cycle. The linear correlation coefficient of $E M_{\mathrm{Q}}^{\mathrm{TOT}}$ and $E M_{\mathrm{F}}^{\mathrm{TOT}}$ is 0.89 , corresponding to a probability smaller than $0.1 \%$ that the two quantities are uncorrelated. The variations 


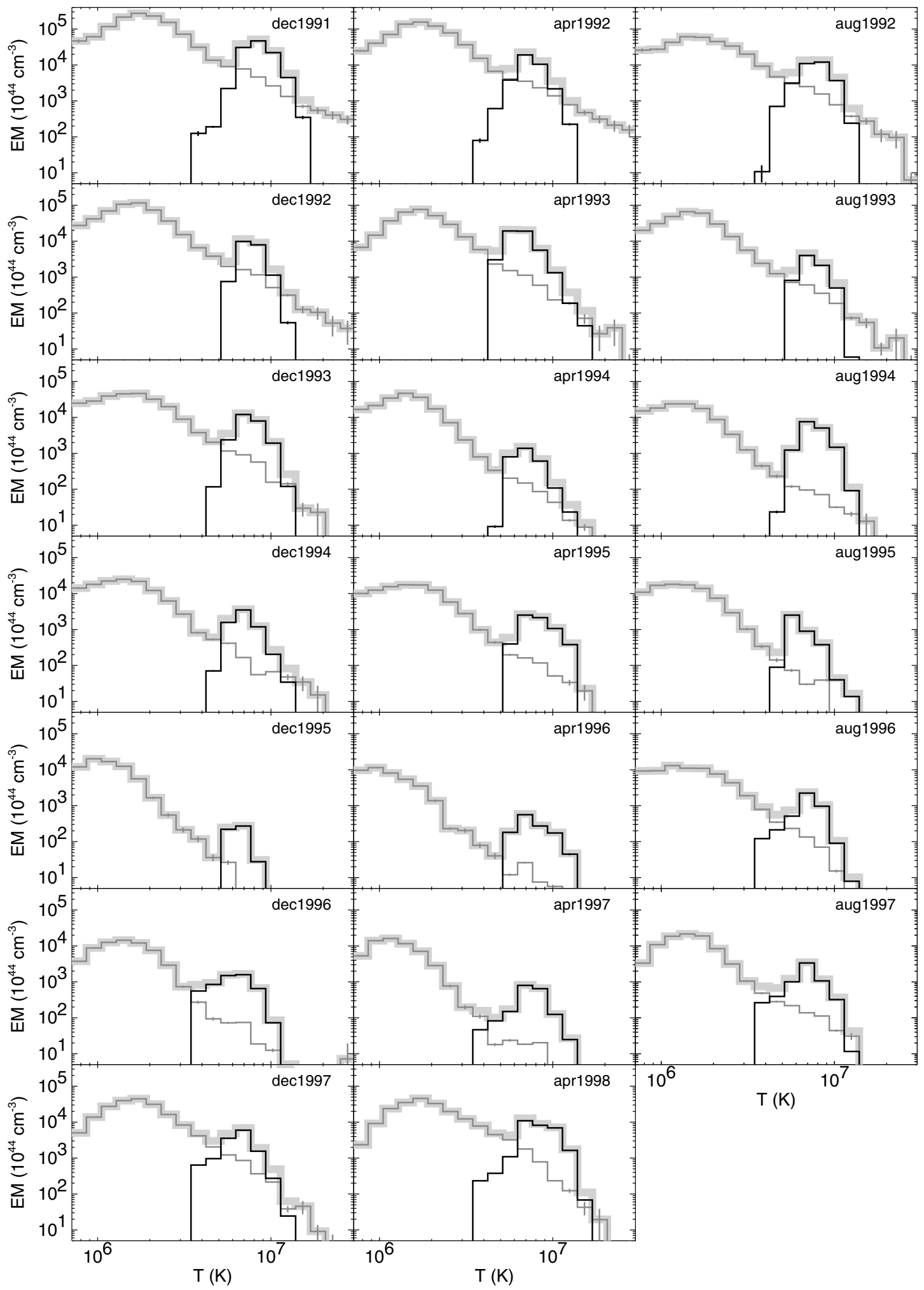

Fig. 4. Emission measure distribution versus temperature of the quiescent (thin line, dark gray) and flaring (thin line, black) solar corona in different months, and their sum (thick line, light gray). Error bars are plotted for each bin, but they are sufficiently large to be visible only for some of the bins.

in $E M_{\mathrm{O}}^{\mathrm{TOT}}$ are smooth over timescales of a few years. Conversely $E M_{\mathrm{F}}^{\mathrm{TOT}}$, superimposed on the modulation due to the solar cycle, shows large variations also on timescales of a few months. Independently of these short timescale fluctuations, the flare component at any time dominates the quiescent emission measure distribution over the range $T \sim 5-10 \mathrm{MK}$ (see Fig. 4).

We repeated the evaluation of the total emission measure of both flaring and quiescent plasma, $E M_{\mathrm{O}}^{\mathrm{HOT}}$ and $E M_{\mathrm{F}}^{\mathrm{HOT}}$, summing only the $E M$ in the range $T \geq 3 \mathrm{MK}$, a temperature range 


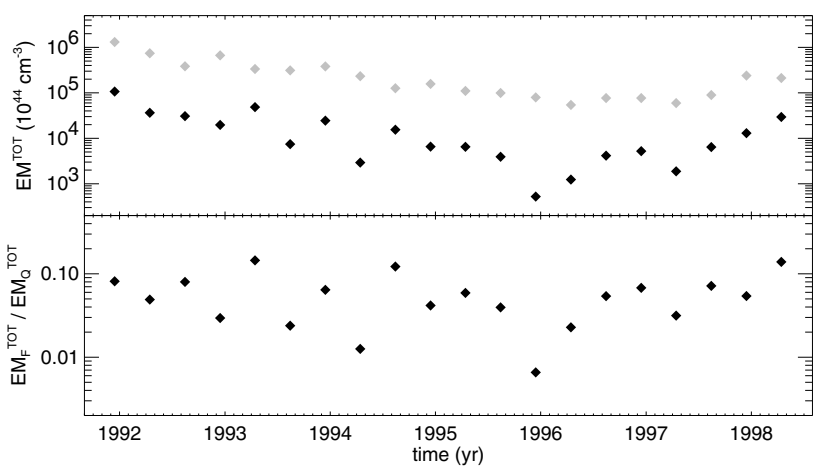

Fig. 5. Upper panel: total emission measure of the flaring $\left(E M_{\mathrm{F}}^{\mathrm{TOT}}\right.$, indicated in black) and quiescent ( $E M_{\mathrm{O}}^{\mathrm{TOT}}$, in gray) coronal plasma vs. time. Lower panel: ratio between total emission measure of flaring and coronal plasma vs. time.

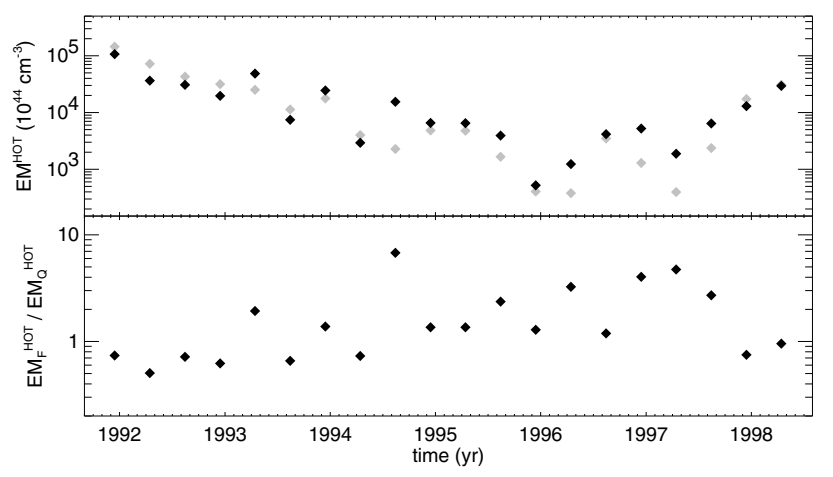

Fig. 6. Upper panel: total emission measure for $T \geq 3 \mathrm{MK}$ of the flaring $\left(E M_{\mathrm{F}}^{\mathrm{HOT}}\right.$, in black) and of the quiescent $\left(E M_{\mathrm{Q}}^{\mathrm{HOT}}\right.$, in gray) coronal plasma vs. time. Lower panel: ratio between emission measure for $T \geq 3 \mathrm{MK}$ of flaring and coronal plasma vs. time.

where both the Yohkoh/SXT and GOES/XRS are well sensitive (Fig. 6). Both components display a variation on timescales of a few years correlated with the solar cycle. They both show variations on shorter timescales (a few months). Their linear correlation coefficient is 0.94 , corresponding to a probability $<0.1 \%$ that $E M_{\mathrm{Q}}^{\mathrm{HOT}}$ and $E M_{\mathrm{F}}^{\mathrm{HOT}}$ are uncorrelated.

The ratio between $E M_{\mathrm{Q}}^{\mathrm{HOT}}$ and $E M_{\mathrm{F}}^{\mathrm{HOT}}$ appears to be slightly larger close to the solar minimum than during the solar maximum, suggesting that the relative contribution of flaring plasma with respect to the hot part of the quiescent corona tends to increase close to solar minimum.

\section{Discussion and conclusions}

The main result of our study is that an unbiased and large sample of solar flares produces an emission measure distribution $E M_{\mathrm{F}}(T)$, time-averaged over a period of one month, that peaks at $T \approx 6-8 \mathrm{MK}$ and is significantly larger than the quiescent emission measure distribution for $T \sim 5-10 \mathrm{MK}$. These properties of $E M_{\mathrm{F}}(T)$ hold irrespective of the solar cycle phase.

Summing $E M_{\mathrm{F}}(T)$ and $E M_{\mathrm{Q}}(T)$, we measured for the first time the time-averaged $E M(T)$ of the entire solar corona, taking into account the average effect of solar flares and quiescent structures. The total solar $E M(T)$ is double peaked, with the cooler peak, at $T \sim 1-2 \mathrm{MK}$ being attributed to the quiescent corona, and the hotter peak, at $T \sim 6-8 \mathrm{MK}$, to the flares.

We also investigated how the quiescent and flare components of the solar corona vary during the solar cycle. The upper panel of Fig. 6 illustrates how the hot components $(T>3 \mathrm{MK})$ of the quiescent and flaring emission measure, $E M_{\mathrm{F}}^{\mathrm{HOT}}$ and $E M_{\mathrm{Q}}^{\mathrm{HOT}}$, vary in a similar way on timescales of a few years following the solar cycle; they are also, however, strongly correlated on shorter timescales of a few months. This implies that the amount of flaring plasma is highly correlated with the hot quiescent structures. The hot tail of quiescent emission measure distribution is caused by the active regions and their cores (Orlando et al. 2001). The observed correlation between flares and active regions may imply that flares occur in active regions (e.g. Bornmann \& Shaw 1994).

The results obtained for the total $E M(T)$ provide new insight into the study of the solar corona heating. It was proposed that coronal heating is caused entirely by flares, ranging from infrequent giant flares to very frequent nanoflares (Parker 1988; Hudson 1991; Klimchuk 2006). In this scenario, both the quiescent and flare components of the solar corona are related to flaring activity; $E M_{\mathrm{F}}(T)$ obviously reflects the high energy tail of the flare distribution, while the plasma contributing to the $E M_{\mathrm{Q}}(T)$ is due instead to less energetic flares (i.e., nano and microflares).

Generally a corona composed of identical loops heated by nanoflares has an $E M(T)$ characterized by one sharp peak (Cargill 1994; Cargill \& Klimchuk 2004). The $E M(T)$ peak is approximately located at the temperature for which the radiative and conductive cooling time, $\tau_{\mathrm{r}}$ and $\tau_{\mathrm{c}}$, are equal. These two characteristic times, and hence the peak temperature, depend on the loop geometry and plasma density. Therefore, a possible explanation of the double peaked $\operatorname{EM}(T)$ of the solar corona is that two different loop distributions exist; this was the case for the theoretical model explored by Güdel (1997), who obtained a double-peaked $\operatorname{EM}(T)$ by studying the effect of a power-law flare distribution, $\mathrm{d} N / \mathrm{d} E \propto E^{-\alpha}$, on loops with different cross sections. However, in the solar corona no evidence was found that major flares occur preferentially in a particular loop class.

Parenti et al. (2006) showed, however, that the $E M(T)$ peak temperature can also be varied by changing the average flare energy. Therefore a double-peaked $E M(T)$ can also arise from two different distributions of flares at different energies. This latter interpretation agrees with the idea that nanoflares and flares are not two portions of the same distribution.

In the present work, we find that the solar total $E M(T)$ has two ever-present distinct peaks, one due to the quiescent corona and one to flares. This simple finding has an important two-fold implication: if we accept the hypothesis that nanoflares heat the quiescent corona, major flares and nanoflares belong to different populations; if we instead assume that flares and nanoflares belong to the same population, the implication is that nanoflares alone cannot explain the emission measure of the quiescent corona.

Beyond the understanding of coronal heating, the results obtained for the total $E M(T)$, flaring plus quiescent, provide new insight into the link between the solar corona and the coronae of active stars. The emission measure distribution $E M(T)$ is a useful means with which to compare coronal plasmas. Active stellar coronae have an emission measure distribution that has a significant peak at $T \sim 8-20 \mathrm{MK}$. Conversely, in the solar corona a significant amount of emission measure at these temperatures is observed only during flares, while quiescent structures have temperatures of a few MK. Starting from this consideration, it was proposed that the hot peak in the $E M(T)$ of active stellar coronae could be due to the presence of a large number of unresolved solar-like flares (e.g. Güdel et al. 1997). 


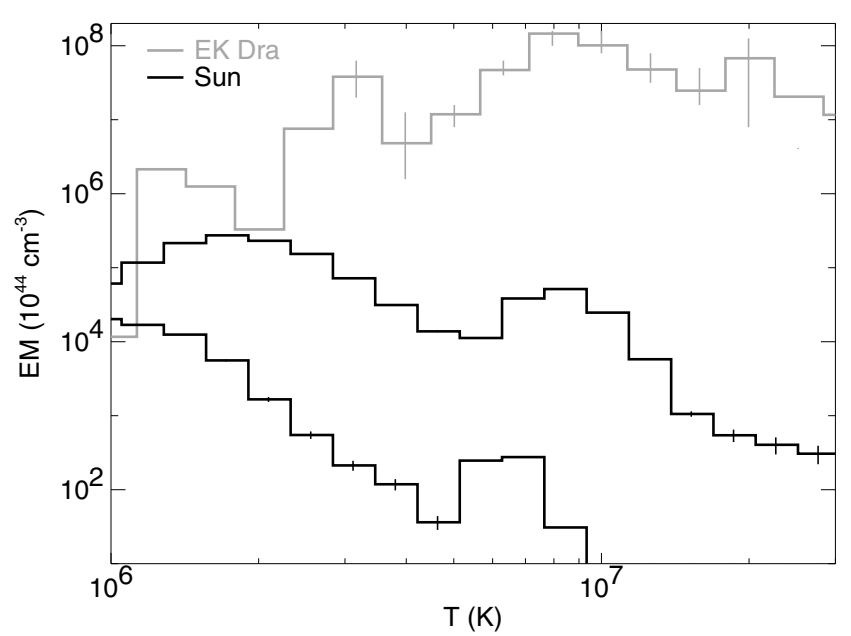

Fig. 7. Solar emission measure distributions (flaring + quiescent) close to the minimum and maximum phases of the solar cycle (December 1991 and December 1995, both in black) compared with the emission measure distribution of EK Dra (Scelsi et al. 2005, in gray). We show error bars in the EK Dra distribution only for those temperature bins where the $E M$ values are constrained.

The results for $E M_{\mathrm{F}}(T)$ and $E M_{\mathrm{Q}}(T)$ are analogous to those for the coronae of active stars. Active stars display a doublepeaked $E M(T)$ and we showed that this also occurs for the solar corona. The solar $E M_{\mathrm{F}}(T)$ is sharply peaked at temperatures (6-8 MK), which are similar, albeit slightly cooler, to the temperatures of active coronae $E M(T)$ peak $(8-20 \mathrm{MK})$. Therefore, it is more plausible that the hot peak of active coronae, in analogy with the solar case, is due to unresolved flares. We note that active stellar flares are on average hotter than the solar flares.

As an example in Fig. 7, the solar emission measure distribution, close to the maximum and minimum phases of the solar cycle, is compared with the distribution of EK Dra (Scelsi et al. 2005), which is a young Sun-like star, a $1.1 M_{\odot}$ star that has newly arrived on the main sequence, with $L_{\mathrm{X}} \sim 10^{30} \mathrm{erg} \mathrm{s}^{-1}$. Inspecting the two solar $E M(T)$, we note that the hot peak is located at higher temperatures close to solar maximum, which is mainly due to the hot part of $E M_{\mathrm{Q}}(T)$ that changes slope with the solar cycle (see Fig. 4). More frequent solar-like flares would enhance $E M_{\mathrm{F}}(T)$ to reproduce the hot peak of the emission measure distribution of active stellar coronae ${ }^{3}$. On the other hand, for the solar plasma to match the $E M(T)$ of active stars, the $E M(T)$ of the quiescent plasma should undergo a smaller increment with respect to that of the flaring plasma. A different scaling of the quiescent and flaring plasma components would then be required to explain the stellar activity.

Based on the assumption that the heating of the corona is due entirely to flares, we inferred that for the Sun either the flare distribution, or the confined plasma response to flares, or both, are bimodal. Comparing the solar $E M(T)$ with that of active stars and considering how the $\operatorname{EM}(T)$ shape changes with activity level, we inferred that the flare and quiescent components have different relative strengths at different stellar activity levels. This result implies that different parts of the flare distribution (i.e. flares, micro- and nano-flares) or the confined plasma response to flares and nano-flares, scale in different ways with stellar activity.

3 It is true if we assume that each flare occurs in a different coronal loop.
A possible scenario in this perspective is that of Drake et al. (2000), who argued that for increasing coronal activity, the large surface coverage of active regions and strong magnetic fields could provoke a change in the dissipation of magnetic energy, favoring more magnetic interactions and reconnections, and therefore a more efficient production of flares. However, this scenario is not supported by the small filling factors inferred for the active stellar coronae (Testa et al. 2004a; Ness et al. 2004).

In conclusion, this work shows that taking into account both flaring and quiescent emission leads to a bimodal emission measure distribution, yielding insight into stellar coronal physics; on the other hand, this work provides a constraint on the distributions of nano-, micro-, and macro-flares.

More precise estimations of the solar corona $\operatorname{EM}(T)$ will be possible with new instruments that provide higher spatial resolution and more accurate temperature estimation, for instance the X-ray telescope (XRT) onboard Hinode (Kosugi et al. 2007), or the Atmospheric Imaging Assembly (AIA) and the Extreme Ultraviolet Variability Experiment (EVE) both onboard the forthcoming Solar Dynamics Observatory (SDO). Hinode/XRT collects X-ray images of the entire disk of the Sun, with $1^{\prime \prime}$ angular resolution, in 9 different bands, allowing a detailed thermal characterization of the entire corona and an excellent derivation of the coronal $E M(T)$. SDO/AIA will gather images of the entire solar corona, in several narrow-band filters, with high spatial resolution ( $\left.1^{\prime \prime}\right)$ and high sampling rate $(\sim 10 \mathrm{~s})$. Thus, SDO/AIA will enable the entire coronal $E M(T)$ (flaring + quiescent) to be derived for a wide range of temperature using only one instrument. SDO/EVE will gather disk-integrated X-ray and UV spectra. Thus, the derived solar $E M(T)$ can be compared with the stellar $\operatorname{EM}(T)$, derived from disk-integrated spectra.

\section{Appendix A: Quiescent contribution estimation in the GOES/XRS data}

To complete the correct analysis of GOES/XRS data, it was important to identify the level that corresponded to the quiescent coronal emission in the one-month-long GOES/XRS light curves in the soft and hard bands. Since the coronal quiescent emission may change over the period of one month, we divided the two GOES/XRS light curves into segments of $24 \mathrm{~h}$, and we assumed that the quiescent emission did not vary in each $24 \mathrm{~h}$ subinterval. We assumed that in each of these $24 \mathrm{~h}$ sub-intervals the observed minimum values of the GOES/XRS fluxes was the quiescent emission. Since however the minimum fluxes of the soft and hard bands usually occurred at different times, we required that the soft and hard fluxes, representing the quiescent emission, should be simultaneous, and not significantly larger than their absolute minimum values. Therefore, we adopted the following procedure: 1) we identified the minimum flux in the hard band; 2) we selected all bins for which the hard flux ranged between its minimum value and its minimum plus an increase of $10 \%$; 3 ) among these bins, we selected one (corresponding to the time $\left.t_{\min }\right)$ for which the soft fluxes is minimum; 4) the hard and soft fluxes recorded at $t_{\min }$ were considered to be representative of the quiescent emission for the $24 \mathrm{~h}$ sub-interval. For each $24 \mathrm{~h}$ interval, we subtracted the relevant quiescent fluxes from the soft and hard fluxes. This procedure for the search of the minimum was applied to the GOES/XRS light curves slightly smoothed (over a time period of $\sim 30 \mathrm{~s}$ ), to avoid problems related to small number statistics. 
Acknowledgements. C.A., G.P., S.O., and F.R. acknowledge partial support for this work from contract ASI-INAF I/023/05/0 and from the Ministero dell'Università e della Ricerca.

\section{References}

Argiroffi, C., Maggio, A., \& Peres, G. 2003, A\&A, 404, 1033

Bornmann, P. L., \& Shaw, D. 1994, Sol. Phys., 150, 127

Cargill, P. J. 1994, ApJ, 422, 381

Cargill, P. J., \& Klimchuk, J. A. 2004, ApJ, 605, 911

Drake, J. J., Peres, G., Orlando, S., Laming, J. M., \& Maggio, A. 2000, ApJ, 545, 1074

Favata, F., \& Micela, G. 2003, Space Sci. Rev., 108, 577

Garcia, H. A. 1994, Sol. Phys., 154, 275

Güdel, M. 1997, ApJ, 480, L121

Güdel, M. 2004, A\&AR, 12, 71

Güdel, M., Schmitt, J. H. M. M., Benz, A. O., \& Elias, II, N. M. 1995, A\&A, 301,201

Güdel, M., Guinan, E. F., \& Skinner, S. L. 1997, ApJ, 483, 947

Hudson, H. S. 1991, Sol. Phys., 133, 357

Klimchuk, J. A. 2006, Sol. Phys., 234, 41

Kosugi, T., Matsuzaki, K., Sakao, T., et al. 2007, Sol. Phys., 243, 3

Marino, A., Micela, G., Peres, G., \& Sciortino, S. 2003, A\&A, 407, L63
Masuda, S., Kosugi, T., Hara, H., Tsuneta, S., \& Ogawara, Y. 1994, Nature, 371, 495

Ness, J.-U., Güdel, M., Schmitt, J. H. M. M., Audard, M., \& Telleschi, A. 2004, A\&A, 427, 667

Orlando, S., Peres, G., \& Reale, F. 2000, ApJ, 528, 524

Orlando, S., Peres, G., \& Reale, F. 2001, ApJ, 560, 499

Orlando, S., Peres, G., \& Reale, F. 2004, A\&A, 424, 677

Parenti, S., Buchlin, E., Cargill, P. J., Galtier, S., \& Vial, J.-C. 2006, ApJ, 651, 1219

Parker, E. N. 1988, ApJ, 330, 474

Peres, G., Orlando, S., Reale, F., Rosner, R., \& Hudson, H. 2000, ApJ, 528, 537

Peres, G., Orlando, S., \& Reale, F. 2004, ApJ, 612, 472

Raassen, A. J. J., Ness, J.-U., Mewe, R., et al. 2003, A\&A, 400, 671

Reale, F., Peres, G., \& Orlando, S. 2001, ApJ, 557, 906

Sanz-Forcada, J., \& Micela, G. 2002, A\&A, 394, 653

Sanz-Forcada, J., Brickhouse, N. S., \& Dupree, A. K. 2002, ApJ, 570, 799

Sanz-Forcada, J., Brickhouse, N. S., \& Dupree, A. K. 2003, ApJS, 145, 147

Scelsi, L., Maggio, A., Peres, G., \& Pallavicini, R. 2005, A\&A, 432, 671

Testa, P., Drake, J. J., \& Peres, G. 2004a, ApJ, 617, 508

Testa, P., Drake, J. J., Peres, G., \& DeLuca, E. E. 2004b, ApJ, 609, L79

Testa, P., Drake, J. J., Peres, G., \& Huenemoerder, D. P. 2007, ApJ, 665, 1349

Tsuneta, S., Acton, L., Bruner, M., et al. 1991, Sol. Phys., 136, 37

Vaiana, G. S., Krieger, A. S., \& Timothy, A. F. 1973, Sol. Phys., 32, 81

Walter, F. M., Cash, W., Charles, P. A., \& Bowyer, C. S. 1980, ApJ, 236, 212 